\title{
SINGLE PASS ANTIPROTON BEAM DIAGNOSTICS OF THE CERN PS ACCELERATOR COMPLEX
}

G. Cyvogt, B. Frammery, A. Krusche, R. Maccaferri M. Ruette, G.C. Schneider, E. Schulte.

CERN, Geneva, Switzerland

\section{Surnma fy}

The beam characteristics of every $\bar{p}$ bunch in the transfer lines and the CPS can be acquired by various monitors during a single pass and the information stored. The signals emanate from high sensitivity transformers, pick-ups, secondary emission grids and $\mathrm{TV}$ screens. After fast analysis and treatment the beam intensity, trajectory (positions), shape, transverse and longitudinal distributions are displayed in analog and/or digital form. Multiple shot storage is provided for some of these parameters. This paper describes the monitors and the signal processing, gives the special features of the storage systems, and presents typical results.

\section{Introduction}

Antiprotons which have been stacked and cooled in the accumulator AA are transferred (ia the beam lines TTL2 and TT2 to the CPS ring at $3.5 \mathrm{GeV} / \mathrm{c}$ (Fig. 1). After acceleration to $26 \mathrm{GeV} / \mathrm{c}$ they are ejected via TT70 to the SPS or TT6 to the ISR ${ }^{2}$. The various beam diagnostic monitors which are shown must function for single pass $\bar{p}$ and $p$ beams of intensity

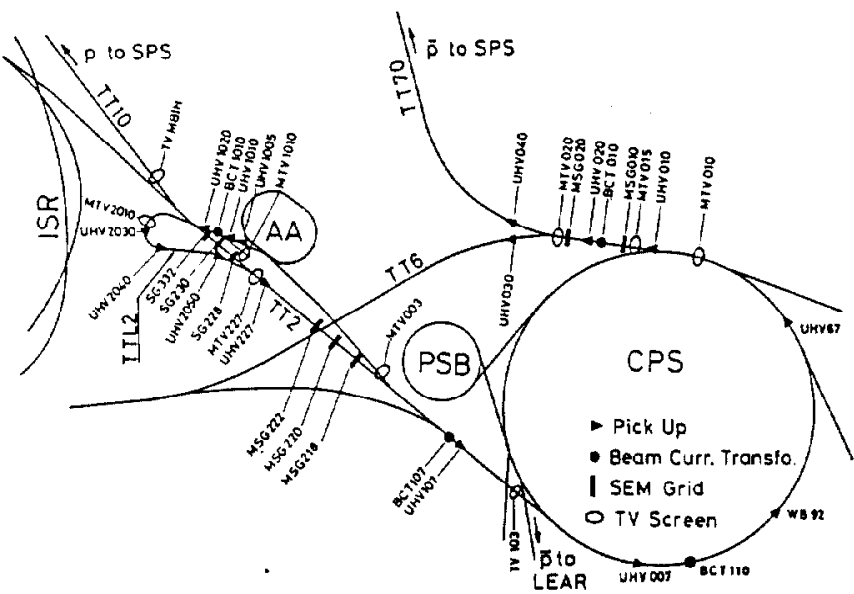

Fig. 1: Monitor Locations for $\bar{p}$ Transfer.

$<10^{9}$ to $10^{12}$ particles per bunch ( $\mathrm{ppb}$ ), bunch length 4 to $100 \mathrm{~ns}$ and position -5 to $+5 \mathrm{~cm}$. All signals must be memorized for later treatment.

\section{Beam Current Transformers (BCT)}

The high resolution of $10^{7} \mathrm{ppb}\left(1 \%\right.$ of $\left.10^{9}\right)$ is obtained by a careful design ${ }^{3}$ ) with the following features: electrostatic and double magnetic shielding of the laminated "U1traperm 10" toroid core, no ceramic chamber (gap located between isolated vacuum flanges), full rotational symmetry (case isolated from support) and symmetrical magnetic shielded transmission cables. Small input noise bandwidth is obtained by a $3^{\text {rd }}$ order resonance circuit (Fig. 2a) which serves also as passive signal integrator. It can be shown that the amplitude $A$ of the output voltage $U(t)$ is proportional to the integrated beam current $I_{B}$ if $T \ll T$ (Fig. $\left.2 b\right)$. With properly chosen elements the integration can then be replaced by peak detection. This method avaids the usual problems with fast, active integrators.
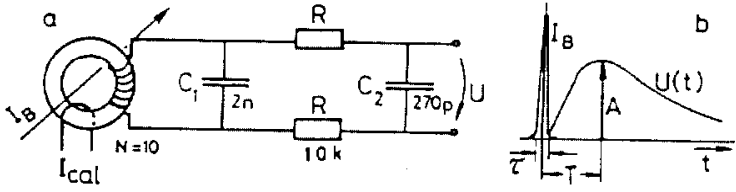

Fig. 2: a) BCT + Filter, b) In and Output Signals

The output signal $U$ is amplified, with a low noise differential amplifier ( $1 \mathrm{nV} / \sqrt{\mathrm{Hz}})$, peak detected, further stretched by a sample and hold ( $\mathrm{S} \& \mathrm{H}$ ) and then digitized by an ADC (Fig. 3).

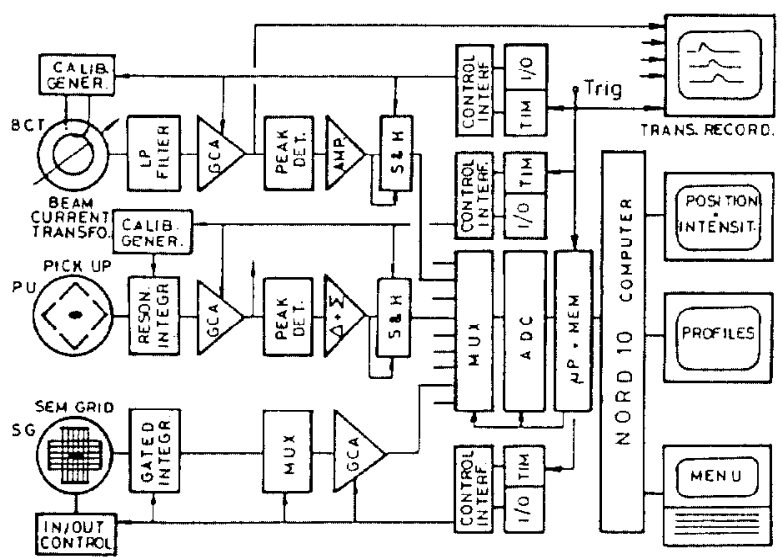

Fig. 3: Beam Diagnostic of BCT, PU and $S G$

Analog signals are available via a transient recorder Typical analog signals are shown in Fig. 4.

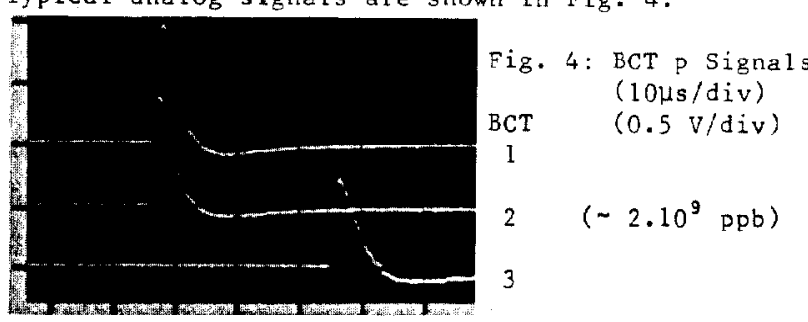

They are used mainly for detection of synchronisation errors in the bean transfers. The digital intensity information is presented as BCT $1,2,3$ (Fig. 5, right).

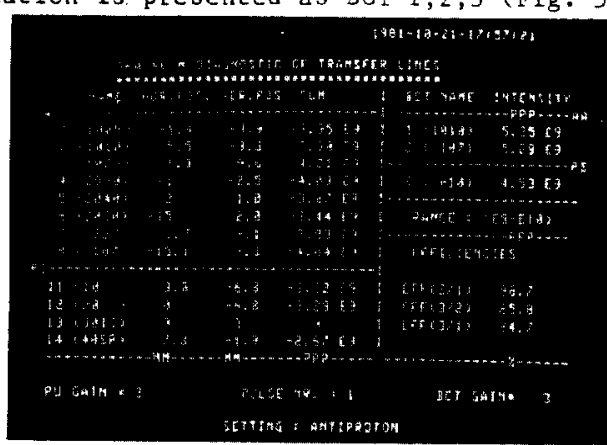

Eig. 5: $\overline{\mathrm{p}}$ Beam Trajectory + Intensity Display

Calibration is obtained by discharge of a cable through a transformer calibration winding in steps of $10^{B}, 10^{9}$, $10^{10}$ or $10^{11}$ particles/pulse (100 ns) and can be performed at any time. 


\section{Pick-Ups (PU)}

The electrostatic PU's are of the combined type shown in Fig. 6 and $7^{3}$.
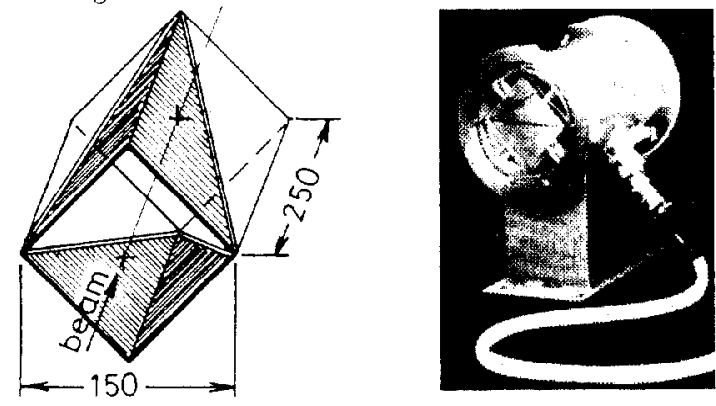

Fig. 5: Pick-Up Electrodes Fig. 7: Pick-Up Station

The horizontal and vertical electrode pairs (stainless steel) together form a straight prism and if correctly sereened have, simultaneously, perfect linearity in both planes ${ }^{4}$ ). This configuration, compared with diagonally cut cylinders, gives better beam intensity and position resolution due to smaller capacity and higher position sensitivity. The input circuit connected co each electrode is a $2^{\text {nd }}$ order low pass filter shown in Fig. 8.

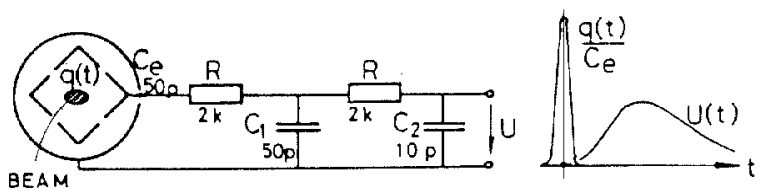

Fig.8: Pick-Up Filter + In and Output Signals

l'his filter decreases the dynamic range due to different bunch lengths $(4 \div 100$ ns $)$ from 25 : I to $1.3: l$ and stretches all pulses to about $250 \mathrm{~ns}$ length. After gain controlled amplification the signals are fed to bipolar peak detectors ${ }^{3}$ ). The sum and difference signals are kept in sample and holds (self triggered via comparator) and sent through a common multiplexer to a CAMAC ADC (Fig. 3). A micro processor controls the acquisition procedure, performs the necessary multiplication with the calibration factors, does the normalization and of fset correction before displaying the beam positions by an event triggered software routine ${ }^{3)}$. The complete trajectories and intensities are stored for the last 24 shots in a push down memory. Fig. 5 (left) shows the horizontal and vertical beam positions in 13 pu's of a $\bar{p}$ transfer to the SPS. Previous trajectories (Pulse No. 2 to 24) can be presented for comparison. A calibration generator can simulate beams of 3 intensilies, 3 positions and 2 polarities ( $\bar{p}$ and $p$ ).

The position resolution of the system is about 0.1 $\mathrm{mm}$ at $10^{20} \mathrm{ppb}$.

\section{SEM Grids (SG)}

For transverse emittance measurements of low intensity $\bar{p}$ beams special secondary emission grid triplets are used. Fifteen $10 \mathrm{wm}$ thick titanium strips of $1 \mathrm{~mm}$ width and $1.5 \mathrm{~mm}$ center spacing cover the cross-section horizontally and a further 15 vertically. The current generated on each strip by the traversing beam is integrated by 30 gated active iutegrators located $200 \mathrm{~m}$ away from the SG (Fig. 9).

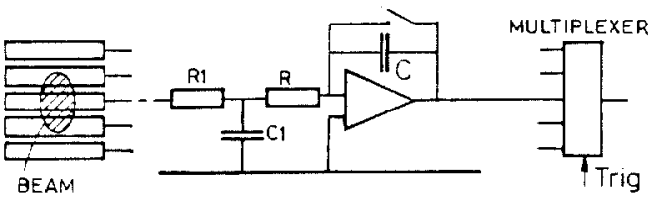

Fig. 9: SEM Grid Integrator
The capacitance $C$ can be adapted to the beam intensity via computer control. The integrated output signals are multiplexed, amplified with gain controlled amplifiers and then digitized ${ }^{5}$.

The microprocessor based acquisition system permits 16 consecutive measurements with minimum interval of $20 \mathrm{~ms}$. To eliminate $50 \mathrm{~Hz}$ ripple the measurements are made in multiples of $20 \mathrm{~ms} e . g$. at the same $50 \mathrm{~Hz}$ phase. The difference between two such acquisitions (without and with beam) is offset and $50 \mathrm{~Hz}$ noise free.

Fig. 10 shows the horizontal and vertical beam profiles of a low intensity $\bar{p}$ beam of 80 ns length.

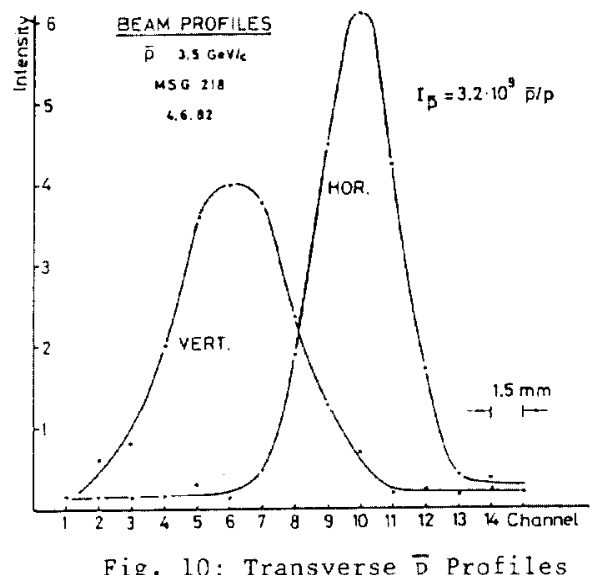

Longitudinal Bunch Shape Measurement

The longitudinal $\bar{p}$ bunch shape, obtained from an electrostatic CPS wideband ( $1 \mathrm{GHz}$ ) pick-up ${ }^{5}$ ), can be acquired lwice per acceleration cycle with $300 \mathrm{~ms}$ interval. Both bunch amplitude and length vary strongly with user requirements. Data from up to 40 bunches can be stored for later analys is in addition to the immediate presentation. The automatic measurement device consists of a programmable fast amplifier/attenuator followed by a Tektronix 7912 A waveform digitizer, driven through a GPIB link by an external 6800 based controller, which performs input amplitude adjustment, trigger selection, data storage and analysis plus analog output display (Fig. 11). The overall bandwidth is $500 \mathrm{MHz}$.

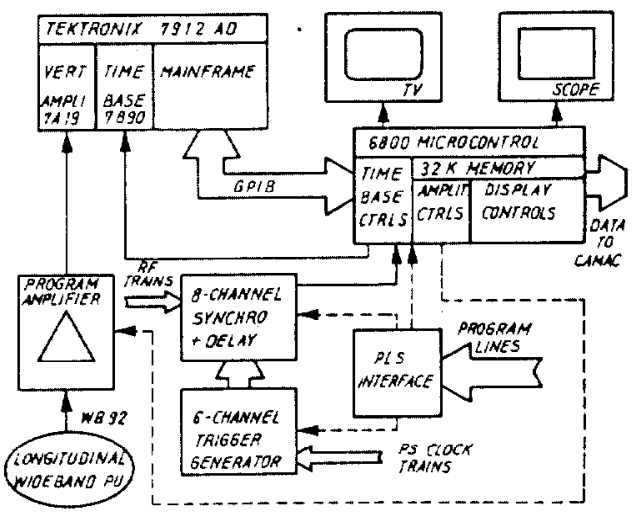

Fig. 11: Longitudinal Bunch Shape Measurement

The triggers are delivered by a multichannel pulse generator which is synchronized to the PS timing. Radio-frequencies serve for fine nanosecond delay adjustment. Every trigger channel is adjusted for a special type of operation and selection is automatically performed according to the PS general Program Line Sequencer (PLS). 
After every trigger, digital data ( $1 \mathrm{~K}$, 9 bits) are transferred to the memory of the micro-controller, checked for validity and stored if the signal peak amplicude is more than $3 \%$ of full scale. New settings are prepared for the vertical input ampliFier, the time base and main-frame of the digitizer. Apart from data collection and digitizer driving, the micro-controller generates two displays (TV and scope) from one of the 40 stored bunch shapes. The first is a video signal including calibrated graticule, record time and time base; the second is a $60 \mathrm{uz}$ repetitive $y-f(t)$ signal for oscilloscope viewing. Fig. 12 shows two $\bar{p}$ bunch shapes of the same bunch a) before and b) after acceleration in the CPS.
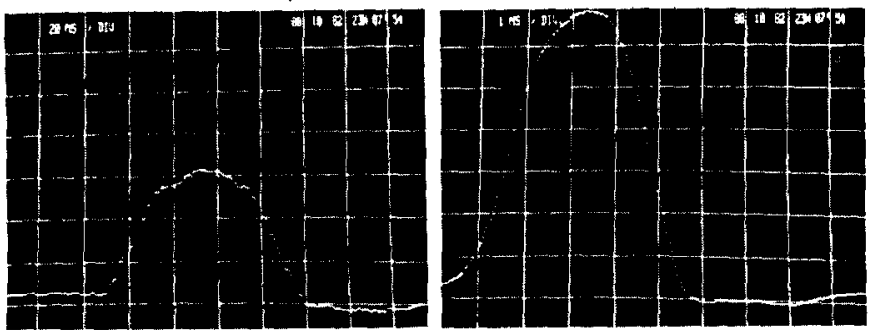

a) $20 \mathrm{~ns} / \mathrm{div}$ (injection) b) I ns/div (ejection) Fig. 12: Longitudinal $\tilde{\mathrm{p}}$ Bunch Shapes

On request, digital data can be transferred to PS computers for long term reference and for longitudinal emittance calculations.

\section{TV Signal Processing}

For the measurement of a single shot bunch with a high sensitivity luminescent screens $\left(\mathrm{AL}_{2} \mathrm{O}_{3}\right)$ having only short remanance time constants, an inexpensive video sample and hold was developed. The principle is shown in Fig. 13.

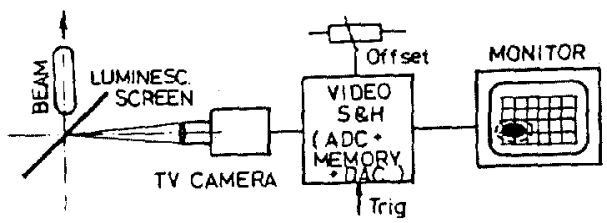

Fig. 13: TV Picture Sample \& Hold

The video signal of the TV camera is digitized by an ADC some milliseconds after the beam has passed the screen. One complete picture (256 × 256 dots) is put into memory and then continuously displayed on the monitor via a DAC.

Fig. 14 shows the block diagram of the video frame sample and hold. The video signal applied to the buffer is split in 3 channels. The first goes directly to the video mixer and then to the monitor. The second is treated (variable gain and offset) to adjust range and level of the signal before it is applied to the ADC. The third is sent to the sync separator.

A store trigger arms the shift register (SRI) and the memory. The next vertical sync pulse starts the acquisition or read procedure. The burst generator, triggered with the horizontal sync pulse, produces 256 clock pulses for the $A D C$ and the shift register (SR1). After 8 clock pulses (via divider $1 / 8$ ) the contents of SRl are loaded into the memory. This is repeated 32 times per line. After 256 lines, the memory contains $8 \mathrm{k}$ bytes and the system switches from the read to the write mode. SR2 is then triggered in a similar way so that the memorized bit sequence is repeated and converted by the DAC. This signal is added to the direct video signal in the mixer. Thus the monitor displays the real time TV screen picture plus, superimposed, a stored previous one.

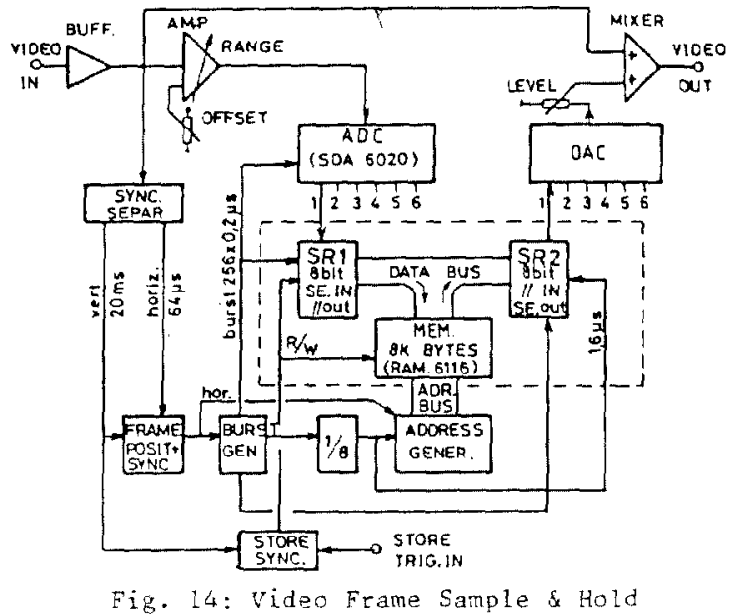

Fig. 15 shows a typical $2 \mathrm{~V}$ picture of a $\overline{\mathrm{p}}$ bunch (10 9 pp) captured with such a device with only 1 bit of the $A D C$ equipped.

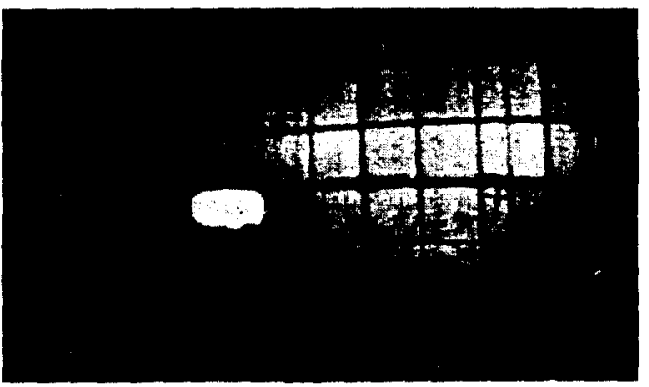

Fig. 15 : Stored $\overline{\mathrm{p}}$ TV Signal

Frequency division $1 / 8$ permits use of slow memories ( $1.6 \mathrm{Ws} c$ lock) leading to the relatively low cost of the device.

\section{Conclision}

Diagnostics of the $p / \vec{p}$ transfers require monicors which combine high sensitivity with high noise immunity and wide dynamic range. Signal processing relies on micro-processor based techniques.

The system has been in operation for about two years. Its performance and reliability allows fast set-up and optimisation of single shot transfers.

\section{Acknowledgements}

We would like to thank out colleagues who have contributed to the systems described here, especially L. Merard, M. Corcelle, M. Martini and P. Pelletier.

\section{References}

1. J. Gareyte, The CERN p-p Complex, Droc. XT International Accelerator Conf. CERN 1980, p.79

2. The PS Staff, The CERN oS Complex as an Antiproton Source, this Conferenre.

3. G.C. Schneider, $\overline{\bar{p}}$ Beam Diagnostics of the Transfer Lines, CERN/PS/BT/83-4.

4. G.C. Schneider, Messwerferfassung und Ubbertragung zum Strahllagebestimmung beim $28 \mathrm{GeV}$ Protonen Synchrotron von CERN, p. 36, thes is 1971, Techn. Univiversität Hannover.

5. J. Dieperink, private commication.

6. H.H. Unstatter, The New Wideband Pick-Up Station of the PS, CERN/PS/SR/70-1 